ISSN: 0212-0267

DOI: http://dx.doi.org/IO.I420I/hedu20I534191205

\title{
LA DOCENCIA FEMENINA \\ EN LA HISPANIA ROMANA: \\ UNA INFUNDADA CONJETURA
}

\section{Women's teaching in the Roman Hispania: Guess unfounded}

Juan José Seguí Marco

Universitat de València. Departamento de Historia de la Antigüedad y de la Cultura Escrita

Correo-e: Juan.j.segui@uv.es

Recepción: 30 de mayo de 20I4. Envío a informantes: 2 de junio de 2014.

Fecha de aceptación definitiva: is de diciembre de 2015

Resumen: En Mérida se conserva una bella estela funeraria dedicada a una joven. Durante largos años se impuso la interpretación de que se trataba de la dedicatoria de una maestra a su discípula y, por tanto, de un ejemplo único y revelador de la docencia femenina en Hispania. Aunque tal punto de vista está hoy descartado todavía se viene filtrando en algunos estudios, de forma más o menos explícita, esta falsa creencia. La realidad es que no existen pruebas de que las mujeres, llevado del caso de algunas situaciones asociadas con la formación de los esclavos, desarrollaran una actividad docente en el ámbito educativo romano. Una circunstancia que se perpetuó con el triunfo del cristianismo. Hispania no fue una excepción en el mundo romano.

Palabras Clave: docencia femenina; educación romana; profesoras en Hispania; mujer enseñante en España romana; escuela romana.

AbSTRACT: In Mérida remains a nice funerary stela dedicated to a young girl. For many years the interpretation was that it means a dedication of a teacher to her disciple and therefore a unique example of women teaching in Hispania was imposed. Although this view is still ruled today remains in various studies that false belief. The reality is that there is no evidence that women, except of certain situations associated with the formation of the slaves, developed an educational activity in Rome. This circumstance remained with the triumph of Christianity. Hispania was no exception in the Roman world. 
KEY words: Female Teaching; Roman education; female teachers in Hispania; Spain Roman woman teacher; Roman school.

$\mathrm{E}$ n el Museo Nacional de Arte Romano de Mérida se conserva una estela funeraria de mármol hallada en la necrópolis romana del sector del Cerro de San Albín, al este de la ciudad, en 1956. Presenta una forma usual para este tipo de piezas: una hornacina moldurada de arco rebajado en forma de edícula, rematada por dos acróteras, enmarcada por sendas pilastras con capiteles pseudocorintios. En la cavidad, aparece esculpido con gran delicadeza el torso de una joven que está tocando un instrumento musical. Se trata de un pandurium, antecedente del laúd y de la guitarra, que la hace un magnífico documento para el conocimiento del instrumental antiguo y, en general, de su música. La calidad del retrato, de una delicadeza pocas veces conseguida en el arte romano provincial, ha contribuido a situarla en un lugar prominente entre las esculturas de la Hispania romana ${ }^{1}$. En la parte inferior de la estela se inserta una inscripción funeraria en la que la fallecida, Lutatia Lupata, una muchacha de I6 años, es recordada por Lutatia Severa como su alumna².

La pieza fue publicada en 1957, un año después de su descubrimiento, por D. Antonio García y Bellido, por aquel entonces catedrático de arqueología en la Universidad Complutense, en el «Noticiario» de una prestigiosa publicación, acompañada de una fotografía y de un dibujo hecho por él mismo de la parte epigráfica ${ }^{3}$ Al realizar el comentario, el autor afirmaba:

El texto nos da a conocer el nombre y la edad de la niña que vemos tocando el laúd: Lutatia Lupata, de dieciséis años cuando le sorprendió la muerte. La dedicante se llamaba como la difunta, Lutatia, y de cognomen Severa. La coincidencia del nomen hace sospechar si la palabra «alumna» ha de tener aquí un sentido más familiar que docente. Yo me inclino, sin embargo, por el segundo, pues en caso contrario se hubiese hecho constar aquel vínculo familiar. Habría que pensar, pues, que Lutatia Lupata era discípula de la dedicante Lupatia Severa, y que ésta, al morir la niña, le dedicó el monumento, en el cual se hizo representar tocando el pandurium. La identidad del nomen habría que atribuirlo, pues, a pura casualidad, o bien a alguna relación no sanguínea; Lutatia Severa pudo haber sido liberta de la familia, aunque es raro, tampoco se haga constar ${ }^{4}$.

Así pues, se proponía, desechando la otra posibilidad, que Lupatia Lupata fuera una discípula de Lupatia Severa que, al fallecer, le dedicó el monumento funerario. Esta propuesta tuvo una amplia acogida dada la autoridad del investigador

Nogales, Trinidad: La mirada de Roma. Catálogo de la exposición, I995, n. ${ }^{\circ}$ I09; EADEM: El retrato privado en Augusta Emerita, Diputación Provincial de Badajoz, I997, n. ${ }^{\circ}$ 69, pp. I03-I05; Caldera de Castro, Pilar: Hispania: El legado de Roma, en el año de Trajano, 1998, n. ${ }^{\circ}$ i7 i, pp. 549 y 603. La ficha de la pieza puede consultarse fácilmente en ceres.mcu.es/pages/Main.

Hispania Epigraphica, 20062: D(is) M(anibus) s(acrum) / Lutatia Lupata, ann(orum) XVI (sexdecim) / Lutatia Severa, alumn(ae) / H(ic) s(ita) e(st). S(ic) t(ibi) t(erra) l(evis).

3 García y Belido, Antonio: «Estela emeritense de Lutatia Lupata», Archivo Español de Arqueología, 30 (1957), pp. 242-243, con inscripción dibujada.

4 García y Belido, Antonio: op. cit., p. 242. 
y del medio en el que se difundió, lo que determinó el rumbo que iba a seguir durante años una parte sustancial de la bibliografía y de la investigación, tanto española como internacional. En efecto, tras una primera puesta en conocimiento en el mundo epigráficos, fue precisamente aquí donde inicialmente se pudo apreciar esta incidencia. Dos obras coetáneas editadas tres lustros después recogían la pieza: una, con carácter recopilatorio de las principales inscripciones romanas de Hispania, la clasificaba entre aquellas que harían mención a personajes con actividades de artes y oficios, en concreto entre las de carácter pedagógico ${ }^{6}$; otra publicación, dedicada a esclavos y libertos hispanos, en la que se utilizaba básicamente material lapidario, la incluía como una prueba de una actividad laboral vinculada al mundo de la música7 .

El paso definitivo en la consolidación de este punto de vista se plasmó en un artículo que, cerca de veinte años después de la noticia inaugural de su descubrimiento, se ocupaba de la enseñanza en la Hispania romana, señero en este sentido, pues se trataba de la primera ocasión en que el tema educativo en la península durante aquellos siglos era abordado directamente y, además, desde una acreditada revista española de historia antigua. Después de hacer una transliteración del texto de García y Bellido, apoyando sus tesis, los autores llegaban aún más lejos y proponían que Lutatia Severa fuese una grammatica, basándose en que la edad de la difunta era la propia de los que cursaban este nivel, resaltando que se trataba de la primera mujer docente que aparecía en las fuentes, la única representante hasta el momento de esta profesión en la península ${ }^{8}$. Un año más tarde los mismos autores insistían en esta misma línea?.

Así pues, durante bastante tiempo, esta interpretación de la estela se mantuvo sólidamente asentada, hasta que tres lustros después se produjo un viraje radical, precisamente por donde menos cabía esperarlo; pues llegó a partir de la rectificación de uno de los defensores de la interpretación aceptada hasta el momento, que pasó a desecharla por completo, admitiendo que se trataba de un caso claro de «alumnado» jurídico -es decir, inclinándose ahora por la vía que descartara García y Bellido-, similar a otros ya constatados en Hispania y que, por consiguiente, no ofrecía ninguna particularidad, ni tan siquiera por el hecho de asumirla una mujer, algo de lo que había también varios ejemplos ${ }^{10}$.

$A E$ (1959), p. 29.

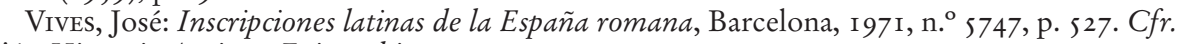
también Hispania Antiqua Epigraphica, I950-1969, n. ${ }^{\circ}$ I 48 I.

Mangas, Julio: Esclavos y libertos en la España romana, Salamanca, I97 I, pp. 91 y i 54 . Algo después la estela se presenta simplemente como una muestra de la afición de la mujer por la música. Vid. Albertos, María Lourdes: «La mujer hispanorromana a través de la epigrafía», Revista de la Universidad Complutense, 26 (1977), pp. I79-198, en concreto, p. I8 I, fig. 2.

8 Sagredo, Luis y Crespo, Santos: «La enseñanza en la Hispania romana», Hispania Antiqua, V (I975), pp. I2 I-I 34, especialmente pp. I27-I 28.

9 SAgredo, Luis y Crespo, Santos: "Las profesiones en la sociedad de Hispania romana», Hispania Antiqua, vi (I976), pp. 53-78, especialmente pp. 59, 69 y 74-75.

ro Crespo, Santos: «El término alumnus indicador de una forma de dependencia personal en Hispania romana», Minerva, 6 ( I 992), pp. 225-239 y 229, nota 28; n. ${ }^{\circ}$ I 3, pp. 232, 235 y 237. Aunque no fue publicada, según parece, Luis GARcía IGLESIAS, en su tesis doctoral, Las inscripciones romanas de Angusta Emerita, Universidad Complutense de Madrid, I973, ya apuntaba este tipo de relación. 
Al poco ya aparecía adecuadamente clasificado al margen de cualquier vínculo de carácter educativo ${ }^{\text {II }}$, y así ha quedado consolidado definitivamente en la moderna investigación ${ }^{12}$.

Y es que los alumni en el mundo romano presentaban una condición de individuos bien definida, inserta dentro de una categoría propia del ordenamiento social, que nada tenía que ver con la docencia. La palabra alumnus no era sinónima de discipulus ${ }^{13}$. Definía, por el contrario, a niños o jóvenes que, sin haber alcanzado la mayoría de edad, se encontraban subordinados a un dominus o patronus que los había recogido y alimentado. En principio, parecía pensada para acoger a esclavos o libertos que hubieran perdido a su propietario o a su patrono y que, por tanto, debían pasar a depender de otro, y que para reflejarlo se les permitía conservar su primitiva onomástica. Pero también se aplicaba a aquellos esclavos o libertos que, sin sufrir este traspaso de propiedad, eran distinguidos con esta designación por su amo o patrono, como una forma de mostrar la predilección y trato diferenciado que hacia ellos se tenía, circunstancia que les aproximaba a un tipo de dependencia especial para un no libre, que corría paralela a la tutela o a la adopción que se podía aplicar a un ingenuo. Estos casos de alumni/alumnae eran a buen seguro muy frecuentes en el mundo romano, ya que cobijaba la práctica frecuente de deshacerse de niños o niñas no deseados ${ }^{14}$.

Así pues, podemos afirmar sin paliativos que Lutatia Severa, quizá una liberta, había recogido a una niña que, como era normal en estas situaciones, se transformó en su alumna, esto es, su protegida. Lupata fue a buen seguro una niña ilegítima, que no habría sido reconocida por el padre, su antiguo amo o un liberto, y que por eso mismo tendría el nombre de la madre ${ }^{15}$. Sin embargo, no debemos pasar por alto que Lupata presenta un cognomen claramente utilizado (lám. 69d).

I2 Nogales, Trinidad: «La imagen del difunto y su evolución en el tiempo», en Edmonson, Jonathan; Nogales, Trinidad y Trillmich, Walter: Imagen y memoria. Monumentos funerarios con retratos en la colonia Augusta Emerita, Madrid, Real Academia de la Historia, 2001, pp. 39-59, fig. I.33 y 2.8, lám. I 2, cat., n. ${ }^{\circ}$ I 2, p. 54; Edmondson, Jonathan: "Catalogue of funerary monuments with portraits», ibidem, pp. I I9-I 87,85 y I45, nota I97.

${ }_{13}$ Thesaurus Linguae Latinae v 6, s. v. discipulus, cols. I327-1329; DE Ruggiero, Enrico: Dizionario epigrafico di Antichità romana, II 3 (I922), s. v. discipulus, p. I9I 2.

${ }_{14}$ Crespo, Santos: op. cit., p. 228; cfr. De Ruggiero, Enrico: Dizionario epigrafico di Antichità romana, I (i895), s.v. alumnus, pp. 437-440. Cfr. Dig. 40, 2, i 2-I4; Smodlaka Kotur, Antonija, «Alumni. Legal Status in Roman Dalmatia (The Dalmatian evidence)», 4 I, I 994, pp. 39 I-4 Io; DESANTI, Lucetta: «Fedecommesso e protezione degli incapaci», Ann. Univ. Ferrara - Sc. Giur, viI (I993), pp. I05-I 24; Boswell, John Eastburn, «Expositio and Oblatio: The Abandonment of Children and the Ancient and Medieval Family», American Historical Review, vol. 89, n. ${ }^{\circ}$ ( (feb., I984), pp. 10-33; Herrmann-Oтto, Elisabeth, «Sklavenkinder in Recht, Ökonomie und Gesellschaft des Römischen Reiches», Revue Internationale des droits de l'Antiquité, 5 I (2004), Nuova Serie vol. (I993), pp. I67I86; Rawson, Beryl: "Children in the Roman familia», en The Family in Ancient Rome, Londres, I986, pp. I73-i 86; Balsdon, John Percy Vyvian Dacre, Roman womens. Their history and habits, Londres, I974, p. I97.

${ }_{15}$ En el caso de que hubiera sido su esclava y la hubiese manumitido, Lupata con dieciséis años no habría sido una completa liberta, sino una Latina Iuniana. Cfr. Edmonson, Jonathan: loc. cit., p. I45; Balsdon, John P. V. D.: loc. cit., p. I45. 
por libertos de Emerita, donde contamos con un Aelius Iustus Lupatus ${ }^{16}$. Se ha apuntado incluso, que Lutatia Severa fuese la nutrix de Lutatia Lupata ${ }^{17}$, si bien es difícil porque esta condición se solía reflejar, como después veremos, y no es el caso $^{18}$. Ahora bien, debemos llamar la atención sobre el hecho de que la condición de liberta de Lutatia Severa no es tampoco inequívoca. Nada impide que fuera libre, quizá una mujer adinerada, casada o viuda, que decidiera proteger de forma especial a una niña, liberta o esclava de su propiedad, y para ello la reconociera en calidad de alumna, dándole su nomen y una esmerada educación, en la que destacó por sus dotes musicales ${ }^{19}$.

Aunque, como hemos visto, desde principios de la última década del siglo xx la estela de Emerita quedaba apartada con rotundidad de cualquier relación con el campo educativo, los efectos de la primera clasificación se han dejado sentir por más tiempo del que hubiera sido de desear, citándose impropia y recurrentemente como una prueba del papel de la mujer en la docencia en la Hispania romana. Y lo más grave, pese a serlo, no ha sido que tal cosa se mantuviera obstinadamente hasta la actualidad de forma más o menos explícita entre algunos investigadores de la Antigüedad ${ }^{20}$, sino que, y esto es aún más lesivo, trascendiera a publicaciones que se desenvolvían dentro de otras disciplinas históricas, contaminando ámbitos como la historia de género ${ }^{2 \mathrm{t}} \mathrm{o}$ como los estudios pedagógicos, no sólo en medios españoles ${ }^{22}$ sino incluso extranjeros ${ }^{23}$.

Ahora bien, llegados a este punto, corregido y descartado sin paliativos en los espacios científicos más acreditados el falso unicum de la maestra emeritense, ¿se desvanece con Lutatia Severa la existencia de esta profesión entre las mujeres romanas de Hispania? Si nos atenemos a los testimonios literarios, la educación

${ }_{16}$ Edmonson, Jonathan: loc. cit., n. ${ }^{\circ}$ 19, pp. 163-165.

${ }^{17}$ Idem, «Commemoración funeraria y contexto social», loc. cit., p. 87.

18 Crespo, Santos: Nutrices en el Imperio Romano. I. Estudio de las fuentes y la prosopografía, Valladolid, 2005.

19 Para un caso de este tipo $c f r$. Weaver, Paul: «Children of Freedmen (and Freedwomen)», en Rawson, Beryl (ed.): Marriage, divorce and children in ancient Rome, Oxford, I991, p. 167 y nota 6.

${ }_{20}$ Pérez Negre, José: «Esclavas, semilibres y libertas en época imperial: aspectos sociojurídicos», en Alfaro, Carmen y Noguera, Alejandro (eds.): Actas del Primer Seminario de Estudios sobre la Mujer en la Antigüedad, Valencia, I997, I37-I60, especialmente I 54-I 5 5, nota I I 5; Medina, Silvia: Mujeres y economía en la Hispania romana. Oficios, riqueza y promoción social, tesis doctoral, Departamento de Historia, Universidad de Oviedo, 20 2, pp. 79 y 89; Hernández Guerra, Liborio: Los libertos de la Hispania romana: situación jurídica, promoción social y modos de vida, Ediciones Universidad de Salamanca, 2013, pp. 74 y I78, presentando planteamientos contradictorios.

${ }_{21}$ GARRIDO, Elisa: Historia de las mujeres en España, Madrid, I 997, p. I06, aunque señalando su carácter de excepción en un ámbito educativo dominado por los hombres; también reitera esta visión errónea Alfaro, Carmen: «La mujer y el trabajo en la Hispania prerromana y romana. Actividades domésticas y profesionales», en Del Val Valdivieso, M. ${ }^{a}$ Isabel (coord.): El trabajo de las mujeres en España. Desde la Antigüedad al siglo XX, Dossier de Mélanges de la Casa de Velázquez. Nouvelle série, 40 (2) (2010), pp. I 5-38, en concreto 20 y nota 38.

${ }_{22}$ BARTOLOMÉ, Bernabé: La educación en la Hispania Antigua y Medieval, Madrid, 1992, p. 84, aunque al menos recoge la otra posibilidad.

${ }_{23}$ StAnley, Farland H. Jr.: "Roman education: Observations on the Iberian Experience», Revue des Études Anciennes, 93 (1991), pp. 299-320, en especial p. 309. 
romana, al igual que la griega, estaba a cargo de maestros, es decir, de hombres ${ }^{24}$. Que el oficio de enseñante les estuviera reservado se debía, sin duda, a la base patriarcal de la sociedad romana. El hombre detentaba la función rectora en la familia (paterfamilias, patria potestas) ${ }^{25} \mathrm{y}$, por derivación, en el Estado. La mujer, reducida al papel de esposa y madre, se debía a la casa y a la crianza ${ }^{26}$. Pero tampoco debemos olvidar que la educación tradicional entre los romanos era entendida como un proceso gradual, que arrancaba desde el mismo nacimiento. Los primeros años, centrados en la alimentación, los cuidados y la formación básica de los hijos, la educatio recaía en las mujeres, que asumían así un papel doméstico iniciático fundamental en el sistema educativo romano, parte esencial, respetada y muy celebrada de su labor matrinalis ${ }^{27}$.

Por consiguiente, la misma traditio otorgaba a la mujer un protagonismo en la enseñanza de los hijos hasta los siete años, que dependían de sus cuidados e instrucción, y que a veces, entre las clases más acomodadas, incorporaba en su auxilio a una nodriza (nutrex), que también podía colaborar, con o sin la madre, en las tareas educativas infantiles elementales ${ }^{28}$. Estas nutrices llegaron a jugar un papel muy importante en esta fase formativa, hasta el punto de que muchas mantuvieron a lo largo de su vida una relación afectiva muy intensa con los niños que habían amamantado, criado y educado, vínculo que se prolongó incluso cuando ya habían alcanzado la edad adulta ${ }^{29}$. Son sin duda estas nutrices las que aparecen mencionadas en las inscripciones con el nombre de educatrices ${ }^{30}$ y a las que los

24 Marrou, Henri-Irénée: Historia de la educación en la Antigüedad, Madrid, I985, pp. 34440I; Bonner, Stanley F.: La educación en la Roma antigua, Barcelona, I984, pp. 9i y ss.

${ }_{25}$ Gardner, Jane F.: Women in Roman law and society, Londres, I987, pp. 5-29; LACY, Walter Kirkpatrick: «Patria potestas», en The Family in Ancient Rome, Londres, I986, pp. I 2 I-I 44; FAYER, Carla: Familia romana. Aspetti giuridici ed antiquari, Roma, I994, pp. I23-289; SAlLER, Richard P.: Patriarchy, property and death in the Roman family, Cambridge, I994, pp. I02-I32. Cfr. BonNer, Stanley F.: loc. cit., pp. 20 y ss.

${ }_{26}$ Treggiari, Susan: Roman marriage. Iusti Coninges from the time of Cicero to the time of Ulpian, Oxford, I991, p. 213; Bonner, Stanley F.: loc. cit., pp. 30-33; Anderson, Bonnie S. y ZINsser, Judith P.: Historia de las mujeres. Una historia propia, 2, Barcelona, I991, p. 587.

${ }_{27}$ Maurin, Jean: «Labor matronalis: aspects du travail fémenin à Rome», en LÉvy, Edmond (ed.): La femme dans les sociétés antiques, Strasbourg, I983, pp. I39 y ss.; Bravo, Gonzalo: «La mujer en la economía de la Hispania romana», en Duby, George y Perrot, Michelle: Historia de las mujeres en Occidente, Madrid, I99I, pp. 579-585, en especial p. 58I.

${ }_{28}$ Corbier, Mireille: «La petit enfance a Rome: lois, normes, pratiques individuelles et collectives», Annales. Histoire, Sciences Socials, 54, 6 (1999), p. I 280.

${ }_{29}$ KAMPEn, Natalie: Image and status: Roman working women in Ostia, Berlin, I98 I, p. I 10; Bradley, Keith R.: Discovering the Roman Family. Studies in Roman Social History, Oxford, I99I, pp. I4-20. Sus textos e inscripciones en EIchenauer, Monika: Untersuchungen zur Arbeits welt der Frau in der römischen Antike, Frankfurt am Main, I988, pp. 246-29I.

30 Thesaurus Linguae Latinae v 2, s. v. educatrix, col. I I3. Inscripciones: CIL v, 3519 (Verona): $D($ is) M(anibus) / C(aesia?) Roma/na Caesiae / l(iberta) educa/tri(ci)f(iliorum) IIII / b(ene) m(erenti); CIL VI, I478: Osciae Modestae M. [f.] / Corneliae Publian[ae] / C. f. I aviae carissimae [et] / educatrici dulcissim[ae] / M. Fl. Arrius Osciu[s] / Honoratus nepo[s] / IIII vir. trib [mil. leg. ---]; CIL vI, 9792: Praecedere volvisti sanctissima coniux / ut me relinqueres in lachrimis / si esta liquit in infernas partes bene / ego autem sine te vitam sordidam exigo / esto felix et ibi dulcissima Thalassia / educatrix c. v. et mibi in convivio per annos XXXX / Papirius Vitalis arte pictoria maritus eius / feminae incomparabili fecit sibi et suis.; CIL VI, I 8848: [M. Cu]ratius / [Zephy]rinus fecit / [Barron]iae Galene / [edu]catrici; CIL VI, 3091 s: Isidi Lydiae / educatrici / Valvas cum / Anubi et ara / Mucianus Aug. / lib. proc.; CIL XIII: 
autores clásicos reconocían sin reparos un papel formativo durante los primeros años de niños y niñas ${ }^{31}$.

A partir de los siete años el padre tomaba el relevo en la educación de los hijos, en especial de los varones. Una parte la asumía personalmente, pero otra la podía delegar en personas capacitadas, algo que fue haciéndose habitual según la sociedad romana perdió su primitiva sobriedad y fue requiriendo una formación más amplia, diversa y compleja. Aquellas familias que se lo podían permitir, especialmente del orden senatorial o ecuestre, dispusieron de paedagogi particulares, pertenecientes sobre todo al grupo de los libertos, algunos tan bien preparados y prestigiosos que eran muy solicitados y a los que se pagaba salarios muy elevados ${ }^{32}$. La inmensa mayoría, no obstante, era simplemente vernae, esclavos nacidos en la casa, que acompañaban a los niños a la escuela (custos) y que les podían ayudar en sus tareas escolares $^{33}$. Su uso se extendió mucho y eso explica la preocupación por que estuvieran bien preparados, siendo el miedo a las malas niñeras y a los pedagogos perniciosos un lugar común en la literatura romana ${ }^{34}$. Como es de imaginar, sólo una minoría se podía sufragar un maestro privado. La mayoría de los niños y las niñas a partir de su séptimo aniversario tenían que ingresar para formarse en una escuela primaria (ludus), que estaba bajo la autoridad de un litterator, primus magister, magister ludi o magister ludi litterariỉs. Las había muy concurridas, aunque otras contaban con muy pocos alumnos, dependiendo de la calidad del maestro y de su sueldo $^{36}$. Al menos, en este estadio primario de la educación, tanto los niños como las niñas podían asistir juntos a clase ${ }^{37}$, un lugar que podía ser un local cualquiera, un simple porche o cobertizo (pergula), un espacio alquilado o la casa del mismo maestro. El programa era sencillo: lectura, escritura y cálculo ${ }^{38}$. Después, de proseguir sus estudios, los niños y las niñas pasaban a la enseñanza secundaria, a cargo de un grammaticus, bien de forma privada o en grupo, en este caso, en una schola ${ }^{39}$. Como ocurría entre los enseñantes de nivel inferior, los más valorados y, por tanto, los mejor remunerados eran los que ejercían la enseñanza privada, como preceptores en familias pudientes, tanto en época republicana como imperial ${ }^{40}$. El estudio

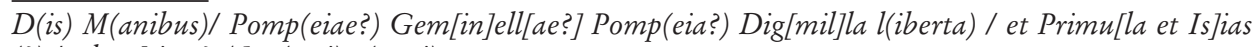
(?) / educt rices? A]ug(usti) n(ostri).

${ }^{31}$ Varro, Logistorici, fr. 5, 1. 2: «Educat obstetrix, educat nutrix, instituit paedagogus, docet magister». La diferencia entre las educatrices y las paegagogae, que después veremos, es subrayada por Eichenauer, Monika: loc. cit., pp. 7-9. Como ejemplo del uso epigráfico en Hispania $c f r$. Hernández, Ricardo y Siles, Jaime: «Nueva lectura e interpretación de una inscripción latina de Villalpando (Zamora)», Studia Philologica Valentina, I4, n. s. I I (20 I 2), pp. 89-97.

32 Bonner, Stanley F.: loc. cit., pp. 40-4I; Bradley, Keith R.: loc. cit., p. 38.

33 Bonner, Stanley F.: loc. cit., pp. 58-64.

34 Sen. De ira, II, 2 I; Quint. I, I, 2-4 y 9; 3, I7; Petron. Sat., 46; Plut., Mor. I, I97. Cfr. Bonner, Stanley F.: loc. cit., pp. I40-I4I y I47.

${ }_{35}$ Marrou, Henri- Irénée: loc. cit., p. 345; Bonner, Stanley F.: loc. cit., pp. 60 y ss.; Galino,

María Ángeles: Historia de la educación. Edades Antigua y Media, Madrid, I988, p. 267.

36 Galino, María Ángeles: loc. cit., p. 264.

37 Bonner, Stanley F.: loc. cit., p. I 82; Galino, María Ángeles: loc. cit., p. 267.

${ }_{38}$ Galino, María Ángeles: loc. cit., pp. 266-267.

39 Marrou, Henri-Irénée: loc. cit., pp. 355 y ss.; Bonner, Stanley F.: loc. cit., pp. 7 I y ss.; Galino, María Ángeles: loc. cit., p. 265.

40 Bonner, Stanley F.: loc. cit., pp. 42-54. 
de la lengua, el comentario de los poetas y las artes liberales (historia, música, astronomía, geometría y matemáticas) centraban el campo formativo ${ }^{41}$. La mayoría eran libertos, si bien los había libres de nacimiento ${ }^{42}$. Por último, de forma mucho más minoritaria, a partir de los diecisiete años, los alumnos más aventajados y con recursos económicos podían cursar los estudios superiores, para lo que se ponían en manos de un rethor ${ }^{43}$. Con la enseñanza de la retórica, de la buena elocuencia, se culminaba la educación romana. Aunque pudiera pensarse que estaba limitada a políticos y abogados, la retórica era estudiada por aquellos que deseaban una selecta educación, pues no sólo formaba en el arte de la elocuencia, sino que alcanzaba materias tales como la historia, la filosofía y la poesía ${ }^{44}$.

Como hemos indicado, las diferencias de ingresos entre los enseñantes eran muy notables en función de su formación y del medio en el que se desenvolvían. El maestro de educación primaria, considerado de baja categoría (rem indignissima), recibía un pobre salario ${ }^{45}$. Sólo en los niveles superiores (grammaticus, rethor), el respeto social y, en correlación, el salario mejoraban, especialmente para aquellos que lo hacían como profesores particulares ${ }^{46}$.

El Estado romano, involucrado en la difusión de la enseñanza entre sus ciudadanos, con independencia de su sexo, intentaba compensar estas deficiencias salariales otorgando a los docentes algunos privilegios de orden fiscal, como la reducción de impuestos o la exención de munera ${ }^{47}$, que debieron contribuir bastante a reforzar la educación pública en los municipios y las colonias del imperio y en sus respectivos territoria, incluidas las pequeñas aldeas (vici), alcanzando su máxima expresión bajo el gobierno de Antonino Pío ${ }^{4}$. El hecho de que a partir de Antonino Caracalla se obligara a los munera a los maestros de primaria -qui pueros primas litteras docent- indica que hasta ese momento éstos quedaban al margen de los trabajos comunitarios, aunque no de la evicción fiscal, que se aplicaría sólo a los docentes de grado superior, si bien con la recomendación a las autoridades de que no se les impusieran cargas por encima de sus posibilidades ${ }^{49}$. Por una epístola de Antonino Pío sabemos que las ciudades menores podían tener hasta tres gramáticos con inmunitas fiscal, mientras que las más grandes podían llegar hasta cuatro. Ventajas que se mantuvieron bajo los Severos, que permitieron destinar una parte de los réditos de los ingresos municipales a los estipendios de los maestros ${ }^{50}$. Más tarde, con los Gordianos y el emperador

${ }_{41}$ Galino, María Ángeles: loc. cit., p. 270.

42 Bonner, Stanley F.: loc. cit., pp. 85-92; Agusta-Boularot, Sandrine: «Les réferences

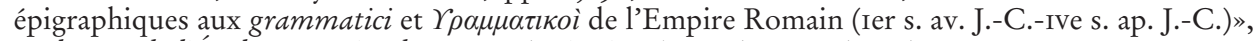
Mélanges de l'École Française de Rome. Antiquités (MEFRA), I06-2 (I994), pp. 653-739.

${ }^{43}$ Marrou, Henri- Irénée: loc. cit., pp. 365 y ss.; Bonner, Stanley F.: loc. cit., pp. 93-Io6.

44 Galino, María Ángeles: loc. cit., pp. 280-282.

45 Galino, María Ángeles: loc. cit., p. 267.

46 Barbagallo, Corrado: Lo stato e l'struzione pubblica nell'impero romano, Catania, I9I I, p. 92.

47 Marrou, Henri-Irénée: loc. cit., pp. 386 y ss.; Bonner, Stanley F.: loc. cit., pp. 2 I 3-2 I 5; GaliNo, María Ángeles: loc. cit., pp. 282-283.

${ }_{48}$ En Dig. 50, 5, 2, 8. Cfr. Barbagallo, Corrado: loc. cit., pp. 47 y 385-366.

49 Dig. 50, 4, i I, 4 y 5, 2, 8. Cfr. Barbagallo, Corrado: loc. cit., pp. 49-50.

so Dig. 27, I, 6, 2. Cfr. Barbagallo, Corrado: loc. cit., pp. I39-175 y 207. 
Probo, se ampliaron estas competencias ${ }^{51}$, hasta cuajar en las regulaciones de Diocleciano, que en su edicto del 301 establecía los honorarios de los enseñantes ${ }^{52}$. La legislación de Constantino confirmaba, y aun ampliaba, todas las medidas protectoras de la enseñanza adoptadas por sus antecesores ${ }^{53}$. El mismo espíritu que se mantiene en emperadores como Juliano y Valentiniano ${ }^{54}$. Una enseñanza, no debemos pasarlo por alto, que en todos los niveles no estaba regulada por programas o métodos detallados, sino que dejaba a los docentes la planificación autónoma de sus enseñanzas, siendo los resultados de los alumnos los que determinaban su validez ${ }^{55}$.

La transformación de la administración del Imperio romano a partir del siglo iv traslada la autonomía municipal en el nombramiento de los docentes, al control superior por parte del Estado. Los gobernadores provinciales y los prefectos del pretorio pasan a supervisar la apertura y la clausura de las escuelas públicas y privadas ${ }^{56}$, el régimen económico y los privilegios de sus maestros ${ }^{57}$ y las condiciones de los estudiantes ${ }^{58}$. No hemos de pasar por alto que el Estado favoreció políticas complementarias de apoyo a la formación educativa, creando instituciones como los alimenta, establecidos en Italia por Trajano, que, a través de los intereses que aportaban los créditos públicos facilitados a las explotaciones rurales, debían cubrir la educación primaria tanto de niños como de niñas 5 . Sabemos que fueron imitados por otras ciudades fuera de la península italiana, y que sus gastos no sólo iban a cargo del presupuesto municipal, sino que muchas veces corrían por cuenta de filántropos ${ }^{60}$. Es muy ilustrativo recordar el papel de Plinio el Joven como mecenas, junto a otros padres, en el nacimiento de una nueva escuela en su localidad natal de Comum $^{61}$, y la importancia que éste atribuye a la educación en la formación de los niños ${ }^{62}$. Aunque resulta imposible valorar los resultados del sistema educativo romano, todo hace pensar que la capacidad de lectura estuvo al alcance de amplias capas de la sociedad ${ }^{6}$. Desde Augusto tanto el Estado como los mecenas privados extendieron la costumbre de crear bibliotecas públicas, con personal adscrito a ellas, y situadas en edificios anejos a los templos (pórticos), que albergaban colecciones de libros griegos y latinos ${ }^{64}$.

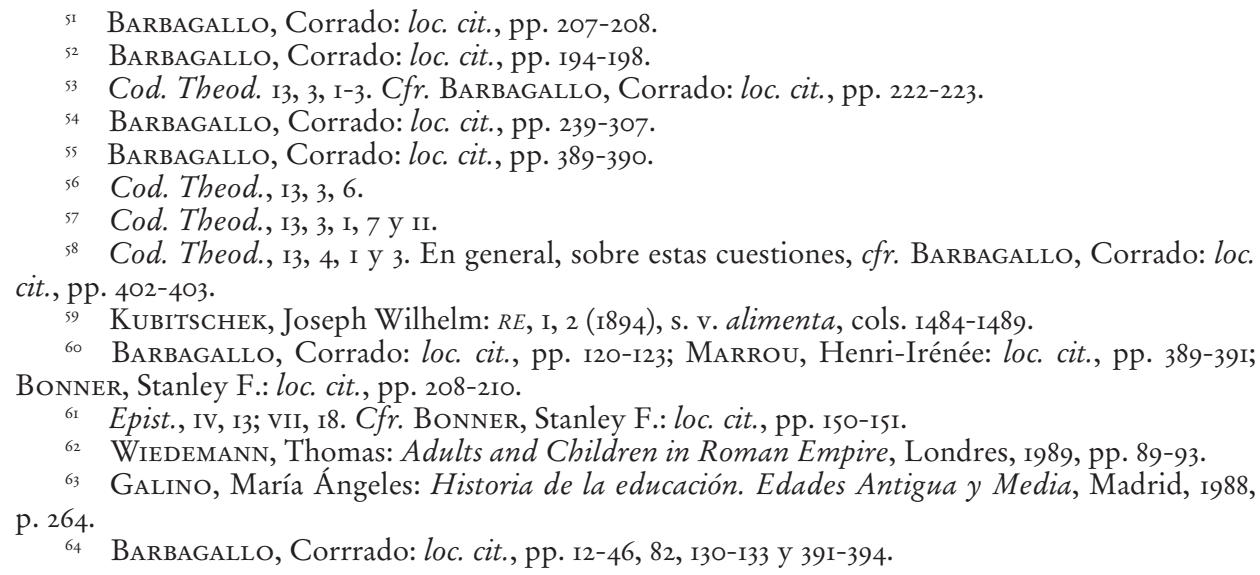


Al quedar relegada la mujer al ámbito laboral doméstico, la norma fue inevitablemente que se la excluyera de todo oficio. Los intelectuales romanos vieron en el hogar el lugar en que debía desarrollarse el trabajo femenino, mientras el masculino estaba en la actividad exterior que, según opinaban, se corroboraba por las diferencias físicas entre ambos sexos, establecidas por voluntad divina ${ }^{65}$. Una mentalidad que se mantuvo de forma constante desde que la consagró Catón, para quien la mejor labor femenina, la que honraba sus tareas como buena esposa, estaba en el tejido de la lana (lanifica) ${ }^{66}$. Pero sabemos que en la práctica esto no fue así. Si la comparamos con la mujer griega, la romana nunca dejó de estar presente en muchos ámbitos profesionales, aunque algunos le fueran expresamente prohibidos por ser propios de los opera virilia ${ }^{67}$. Dotada por el derecho romano de una gran capacidad para administrar sus bienes, con el correr de los tiempos la mujer romana accedió al mundo laboral. Tanto el talento, la preparación y la sagacidad femeninas ${ }^{68}$, como las condiciones reales de la sociedad romana, que andando los siglos poco tendría que ver con los tiempos en que eran reverenciados los mores maiorum más estrictos, arrumbaron las ataduras que la tradición les había impuesto hasta entonces. El desarrollo económico del Imperio romano ayudó, si no fue causa de este proceso, pues provocó desde fines de la República una fuerte demanda de bienes y servicios que alcanzó a vastas poblaciones de los grandes centros urbanos y de las pequeñas comunidades provinciales ${ }^{69}$. En este contexto, las mujeres encontraron grandes posibilidades de incorporarse al mundo laboral, aunque fuera a gran distancia de los hombres, en algunos casos compartiendo con ellos los mismos officia y, en otros, desempeñando tareas específicas. Así, las tenemos como actrices, instrumentalistas musicales, bailarinas, operarias, etc., aunque muy pocas en oficios liberales ${ }^{7}$. Es indudable que de algunas profesiones clave quedaron por completo excluidas como, en general, las que entrañaban responsabilidades judiciales, financieras, políticas o militares ${ }^{71}$.

Pero, ¿y en la profesión de enseñante? ¿ ¿ntervinieron las mujeres en la docencia? Las fuentes de información literaria que poseemos de la enseñanza romana nos hablan siempre de que ésta se encontraba, en todos sus grados, en manos masculinas. Plauto, Cicerón, Horacio, Marcial, Juvenal, Quintiliano, y un largo

65 Collum. XII: Natura comparata est mulieris ad domesticam diligentiam, viri autem ad exercitationem forensem et extraneam. Itaque viro calores et frigora perpetienda, tum etiam itinera et labor paces ac belli, id est rusticationis et militarium stipendiorum dues tribuit: mulieri deinceps quod omnibus his rebus eam fecerat inhabilem, domestica negotia curanda tradidit.

${ }_{66}$ Maurin, Jean: «Labor matronalis: aspects du travail féminin à Rome», en LÉvy, Edmond (ed.): La femme dans les sociétés antiques, Strasbourg, 1983, p. 146.

${ }_{67}$ Dig. 2, I3, I2. Cfr. Del Castillo, Arcadio: «El papel económico de las mujeres en el Alto Imperio Romano», Revista Internacional de Sociología, 32, 9 (1974), p. 60.

68 Iyanga, Augusto y Camprubí, María Inmaculada: La educación de la mujer en la historia, Valencia, 1984, p. 30.

69 FERRER, Juan José: La república participada. Intereses privados y negocios públicos en Roma, Castellón, Universitat Jaume I, 2055, pp. 39 y ss.; GARnsey, Peter y SAlleR, Richard: El imperio romano. Economía, sociedad y cultura, Barcelona, 1990, pp. 63 y ss.

7o Para una relación de oficios femeninos cfr. Eichenauer, Monika: loc. cit., pp. 56-59.

71 Kampen, Natalie: loc. cit., pp. I07-I29; Eichenauer, Monika: loc. cit., pp. 56-59; Bravo, G.: op. cit., p. 582; GARDNER, Jane F.: loc. cit., pp. 237-255. 
etcétera de autores clásicos sólo nos hablan de hombres impartiendo docencia en los tres niveles educativos ${ }^{72}$, máxime cuando los maestros ejercían unos métodos muchas veces cargados de violencia, incompatibles con la condición femenina $^{73}$. Y, en consecuencia, como oficio masculino ha sido presentado por las principales obras de referencia sobre historia de la educación romana ${ }^{74}$. Sin embargo, si desplazamos nuestra atención de las fuentes literarias a las epigráficas nos encontramos con lápidas funerarias donde sí nos aparecen algunas mujeres designadas como paedagoga $e^{75}$. ¿Nos hallamos acaso ante una contradicción entre las fuentes? ¿Es posible entonces que hubiera mujeres al menos en el grado elemental de la educación romana? Cabe señalar, en primer lugar, que estos testimonios de paedagogae en el Imperio romano son exiguos. De los 98 paedagogi identificados epigráficamente, sólo 7 corresponden a mujeres ${ }^{76}$, con la dificultad añadida de que en algún caso resulta imposible saber si estamos ante un hombre o una mujer 7 . Todas ellas aparecen en un mismo contexto. Son libertas o esclavas $^{78}$ vinculadas a familias pudientes. Las tenemos referenciadas entre los Statilii $i^{79}$, los Sulpicii Galbae ${ }^{80} \mathrm{y}$, de forma más dudosa, entre los Volusii ${ }^{8 \mathrm{I}}$, además de hallarlas en otras familias sin identificar, todas de alto nivel ${ }^{8_{2}}$. No se trata de un fenómeno sólo de la ciudad de Roma, como en los ejemplos anteriores ${ }^{83}$. Las

72 Marrou, Henri-Irénée: loc. cit., pp. 360 y ss.; Bonner, Stanley F.: loc. cit., pp. 38 y ss.

73 LEgRAs, Bernard: «Violence ou doucer. Les normes éducatives dans les sociétés grecque et romaine», Histoire de l'éducation, II8 (2008), pp. II-34.

74 Marrou, Henri-Irénée: loc. cit., passim; Bonner, Stanley F.: loc. cit., passim; para ambos el papel educativo de la mujer termina en las nodrizas, y éstas siempre colaborando con paedagogi masculinos; Galino, María Ángeles: loc. cit., pp. 265-273, ni siquiera habla de las nutrices, mientras presenta la educación como una profesión de hombres. Con más detalle, $c f r$. Zaccaria, Claudio: "Paedagoga: un "optional extra?"», en Buonopane, Alfredo y Cenerini, Francesca (eds.): Donna e lavoro nella documentazione epigráfica, Atti del I Seminario sulla condizione femminile nella documentazione epigráfica (Bologna, 2I novembre 2002), Faenza, pp. 23-4I, esp. p. 25 , notas 9-13.

75 Thesaurus Linguae Latinae X I, s. v. paedagoga, col. 34; SCHUPpe, Ernst: RE I8, 2 (1942), s. v. paidagogos, cols. 2375-2385.

${ }_{76}$ ZACCARIa, Claudio: op. cit., pp. 26-27.

77 CIL VI, 4459 (Roma): Philocrate / Messallinae paed(agogus-ae?)/ Accae Helpidis / dec(urionis), en el Monumentum Marcellae. ZacCaria, Claudio: op. cit., p. 26, nota I6, se inclina por un pedagogo masculino.

${ }_{78}$ Eichenauer, Monika: loc. cit., p. I25.

79 CIL VI, 6331: [St]atilia T(iti) l(iberta) Tyrannis / paedagoga / Statiliaes(!). Cfr. Mommsen, Theodor: CIL VI, 2, p. 995 y ZACCARIA, Claudio: op. cit., pp. 3I-33, con abundante bibliografía; LaEs, Christian: «Pegagogues in Latin Inscriptions», Epigraphica, LXXI (2009), pp. 303-325, esp. pp. 3I2 y 320.

8o CIL vI, 9754: C. Sulpicius C(aii) l(ibertus)l / Venustus. / Sulpicia C(aii) l(iberta) Ammia. I Sulpiciae C(aii) f(iliae) Galbillae / paedagogis suis. Según Mommsen, Theodor: CIL vI, 2, p. I275, pedagogo y pedagoga eran hermanos; Sulpicia Galbilla, también liberta, y la dedicante, habrían sido formados por ellos. Cfr. ZACCARIA, Claudio: op. cit., pp. 33-34, con abundante bibliografía; Laes, Christian: op. cit., pp. 315 y 322.

${ }_{81}$ CIL VI, 9756: [---]agae / [--- pae]dagog(ae?) / [posueru?]nt. Cfr. ZaCCARIA, Claudio: op. cit., pp. 34-35, con abundante bibliografía; LaEs, Christian: op. cit., pp. 315 y 322.

${ }_{82}$ CIL VI, 9758: Urbana / paedagoga / v(ixit) an(nos) XXV. Procede de un columbario desconocido. Cfr. Zaccaria, Claudio: op. cit., pp. 35-36; Laes, Chistian: op. cit., pp. 315 y 322.

83 LaEs, Christian: op. cit, pp. 306 y ss. 
encontramos reseñadas, siempre al servicio de importantes familias, en núcleos urbanos de Italia, como en Corfinium ${ }^{84}$, o también en ciudades de provincias, como en Nemausus (Gallia Narbonesis) ${ }^{85}$ o en Thugga (Africa proconsularis) ${ }^{86}$. No hay ninguna duda de que formaban parte de los denominados paedagogia. ¿De qué estamos, pues, hablando? La aristocracia romana y provincial y, desde luego, a la cabeza, la domus Augusta ${ }^{87}$, disponían de escuelas de capacitación para su servicio doméstico y administrativo, organizadas para formar a sus jóvenes esclavos (paedagogiani) bajo la dirección de libertos y esclavos experimentados de ambos sexos (paedagogi/paedagogae puerorum) ${ }^{88}$, que en un mismo paedagogium podían compartir la docencia (compaedagogitae) ${ }^{89}$. Así pues, estas reseñas con que contamos en la epigrafía nos muestran que no estamos ante pedagogas que trabajaran como preceptores privados o maestros públicos en ludi para niños libres (ingenui), sino ante una tarea restringida al mundo servil y muy marginal. ¿Y en los niveles superiores? Al igual que ocurre con la enseñanza elemental, el silencio es absoluto en las fuentes escritas sobre la presencia de la mujer en la enseñanza secundaria romana. Pero de la misma forma que allí nos ocurriera, es la epigrafía romana la que nos presenta un contrapunto. En el África Cesariense tenemos constatada la existencia de una grammatica, de nombre Volusia Tertullina, a la que su marido dedicó una sentida despedida en su inscripción funeraria ${ }^{90}$. Un ejemplo tan excepcional debe ser visto con todo tipo de cautelas. Aunque se ha especulado sobre si se trata o no de un hapax, si estamos o no ante mujer dedicada a la enseñanza o ante una licencia del dedicante, sin poder concluir nada seguro ${ }^{91}$, lo cierto es que un testimonio de este tipo es poco creíble, y cabe pensar que nos hallamos ante una hipérbole marital para definir, en realidad, a una erudita.

Hasta el momento carecemos, en cualquiera de nuestras fuentes, de la más mínima prueba de una presencia femenina en la enseñanza superior. Sí sabemos de mujeres de alta preparación intelectual a lo largo de la época republicana (v. g. Cornelia,

${ }^{84}$ CIL IX, 6325: [---]ec[---] /Benig\{i\}nae / Restitutae / paedago $<g>a$. / Faustus coniux. LAES, Christian: op. cit., pp. 316 y 323 .

85 CIL XII, 3832: D(is) M(anibus)/ Porciae Lade et / Optati ser(vi)/ Epafra conlibert(us) / Syntyche (et) Anatole ser(vae) / paedagogis piissimis / v(ivi) p(osuerunt). Cfr. ZACCARIA, Claudio: op. cit., pp. 38-39; Laes, Christian: op. cit., pp. 317 y 324-325. Cfr. Agusta-Boularot, Sandrine: op. cit., pp. 234235, con pequeñas variantes de desarrollo.

86 CIL VIII, I506: Cornelia / Fortunata / paedagoga / p(ia) v(ixit) a (nnis) LXX/h(ic) [s(ita)e(st)]. Cfr. Zaccaria, Claudio: op. cit., pp. 39-40; Laes, Christian: op. cit., pp. 316 y 323.

${ }_{87}$ ZaCcARIA, Claudio: op. cit., p. 27 y nota 20, con abundante bibliografía. No conservamos, sin embargo, ninguna referencia hasta el momento de paedagogae de la casa imperial.

${ }_{88}$ EnssLin, Wilhelm: $R E$, I8, 2 (I942), s. v. paedagogiani, cols. 2204-2205; Riemann, Hugo: RE, I8, 2 (1942), s. v. Paedagogium Palatini, cols. 2005-2224; Bonner, Stanley F.: loc. cit., pp. 69-70. Cfr. LAEs, Christian: op. cit., p. 305. Para una referencia más sintética cfr. idem: Children in the Roman Empire. Outsiders Within, Cambridge, 20II, pp. II3 y ss.

89 Vid. CIL VI, 633I, 9754 y 9760-9764.

$90 A E$, (1994), n. ${ }^{\circ}$ 58, p. 32 y n. ${ }^{\circ}$ I903, p. 594 de Caesarea (Cherchell): $D$ (is) $M$ (anibus) s(acrum) /Volusiae Tertullinae maritae castae et incomparabili grammat(icae) / quae vix(it) an(nos) XLIII m(enses) III die(s) V, quae cum / marito fecit an(nos) XX m(ensem) I dies XIIII / Domit(ius) Flavianus incompa[(rabili) ---?].

${ }^{11}$ Agusta-Boularot, Sandrine: op. cit., pp. 653-654 y 709-7I2. 
Laelia, Licinia, Sempronia), imperial (v. g. las citadas por Plinio el Joven) o tardorromana (v. g. Hypathia o las que referencia Ausonio), que en las inscripciones aparecen como philosophae o eruditae $e^{92}$, y que sólo tal condición la pudieron lograr muy pocas y en medios muy selectos y restringidos, pero de las que no hay ni rastro de que llegaran a ejercer, ni oficial ni extraoficialmente, la docencia superior.

A la luz de todo lo expuesto, defender la participación de la mujer como docente en la enseñanza de la Hispania romana carece de fundamento, incluso planteada como una simple conjetura ${ }^{93}$. Otra cosa es que la mujer hispana, en especial las que pertenecían a los estamentos superiores y que vivían en el medio urbano, no se beneficiaran de la gran transformación cultural que vivió Hispania cuando la cultura romana se fue extendiendo gradualmente. Recordemos que Estrabón presentaba a la Turdetania como ejemplo de una temprana y profunda romanización ${ }^{94}$ o que Annio Floro describía a Tarraco como una ciudad del más alto nivel cultural, en donde él mismo enseñaba retórica9s. El número de pedagogos masculinos constatados en Hispania no es muy alto, pero suficiente para confirmar, como después veremos, la extensión de la educación en sus tres niveles (paedadogus, grammaticus, rhetor) ${ }^{96}$. Como en todo el Imperio, muchos ejercieron en la península la docencia privada, pero otros lo hicieron a no dudar en la pública, recibiendo honorarios costeados por el municipio. Así se constata en Tritium, donde además sabemos que un grammaticus latinus cobraba un sueldo en la escuela de la localidad durante el siglo II d. C. ${ }^{97}$. Pero la presencia de educadores en Hispania es mucho más antigua, pues se remonta a la época republicana. Hacia el ıoo a. C. Asklepiades de Mirlea enseñaba gramática en la Turdetania ${ }^{98}$. No mucho después, Sertorio fundó una escuela (didaskalia) en Osca para enseñar cultura griega y romana a los hijos de los indígenas más principales y cuyos maestros pagaba el propio general romano99. A mediados del siglo I a. C. había escuelas en la colonia de Corduba, tan concurridas que algunas, como a las que acudió Séneca el Viejo, superaban los doscientos alumnos ${ }^{100}$. La enseñanza primaria se había extendido por Hispania hasta tal punto dos siglos después que en la legislación de un simple distrito minero como fue el de Vipasca (Aljustrel, Portugal), de tiempos de Adriano, se establecía, como si fuera una colonia o municipium romano, la exención de los maestros que allí ejercían de las obligaciones públicas (munera), de las cuales, sin embargo, no estaba excluido el resto de la población, ni siquiera mujeres y niños. De su cumplimiento se encargaba expresamente la máxima autoridad en

92 Agusta-Boularot, Sandrine: op. cit., pp. 736-738.

93 Bravo, Gonzalo: op. cit., p. 582.

94 Strab. III, 2, I5.

95 Grosse, Roberto: Fontes Hispaniae Antiquae viII, 1959, pp. 290-292.

96 Cfr. SAgredo, Luis y Crespo, Santos: op. cit., pp. 53-78.

97 CIL II, 2892: $D$ (is) M(anibus) / L(ucio) Memmio / Probo clu/niensi gram/matico latino / qui res(publica) Tritien/sium an (norum) baben(ti) XXV / salarium constituit / $m$ (ile) c(entum) bic s(itus) $e(s t) s($ it $) t$ (ibi) $t$ (erra) l(evis). Cfr. SAgredo, Luis y Crespo, Santos: op. cit., p. I24.

98 Strab.III, 4, 3.

99 Plut. Sert. I4.

100 Sen. Contr. I; Praef. 2. 
la zona, el procurator metallorum ${ }^{\text {1or }}$. Resulta comprensible esta enorme demanda formativa durante los siglos I y iI d. C. si tenemos en cuenta que la educación se transformó en un trampolín ineludible para la promoción social desde aquel momento. Para las clases alta y media de las provincias romanas resultaba imprescindible una buena formación si se pretendía ejercer un papel político y social relevante en sus ciudades o en el Imperio. La alfabetización era necesaria incluso para las clases bajas en los medios urbanos. Es más, no era posible obtener la ciudadanía, básica para beneficiarse de una condición jurídica plena, si no se sabía latín ${ }^{102}$. La transformación de todas las ciudades de Hispania en municipios romanos o latinos entre Augusto y Vespasiano (74 d. C.) creó, pues, unas perentorias necesidades formativas entre poblaciones de raigambre celta $\mathrm{O}$ ibera que ahora ansiaban adquirir cultura grecorromana. Hispania entró en un proceso casi frenético de latinización acelerada que desbordaba todas sus posibilidades. Las fundaciones alimentarias fueron copiadas por mecenas locales ${ }^{103}$. Nunca hubo en la historia de España un momento como este. Y tardó siglos en repetirse. Al mismo tiempo que el país se llenaba de espléndidos monumentos se requerían escuelas y maestros por doquier ${ }^{104}$. Incluso, en las actividades que realizaban los collegia invenum en las ciudades de todo el Imperio, y que no fueron ajenas a Hispania, se podría intuir algún tipo de formación, aunque sólo fuera de tipo militar ${ }^{105}$. No es exagerado afirmar que la obra de romanización de Hispania, como de todo el mundo romano, sólo fue posible gracias a la escuela municipal ${ }^{106}$.

Este camino ascendente se mantuvo hasta finales del siglo Iv d. C., cuando la situación general se modificó radicalmente a causa de la sustitución del paganismo por el cristianismo. En efecto, a partir de Teodosio I se produjo un cambio muy sensible en lo que se refería a la enseñanza, producto de la reacción antipagana. La inmunidad de los docentes fue limitada por una ley del 383 del Prefecto de Orien$\mathrm{te}^{\mathrm{i07}}$. Aunque las medidas fueron revocadas en esta zona del Imperio a partir del 408, con Teodosio II, no resulta claro que lo fueran en Occidente ${ }^{\mathrm{T} 08}$. La hegemonía cristiana a finales del Imperio reforzó, aunque resulte paradójico, aquellos valores de la sociedad arcaica romana que circunscribían a la mujer al ámbito doméstico, excluyéndola completamente de la enseñanza. Así lo disponen de forma tajante los cánones conciliares de fines de la cuarta centuria, amenazando con severas penas a

ror CIL II, 5I8I, 8 (Lex Vipascensis): Ludi magistri. Ludi magistros a proc(uratore) metallorum immunes es [se concessum est]. Cfr. D’Ors, Álvaro: Epigrafía jurídica de la España romana, Madrid, 1953, p. I03, con comentario.

102 Suet. Cland. I6.

${ }^{103}$ CIL II, I774 (Hispalis). Cfr. SANTERo, José María: Las asociaciones populares en Hispania romana, Sevilla, 1978 , p. I03.

${ }_{104}$ Para la nómina de enseñantes en Hispania, a todos los niveles, $c f r$. SAGredo, Luis y Crespo, Santos: op. cit., 1976, passim. Sólo los gramáticos cfr. Agusta-Boularot, Sandrine: op. cit., pp. 681-683.

ios Barbagallo, Corrado: loc. cit., pp. I8I-I83 y 404-405. Para el papel de estos colegios de jóvenes en Hispania $c f r$. Santero, José María: loc. cit., pp. 97-IO4.

${ }_{106}$ Barbagallo, Corrado: loc. cit., p. 385. Sobre la trascendencia de este proceso en el Occidente romano cfr. Bowen, James: Historia de la educación occidental, I, Barcelona, 1976, pp. 273-276.

${ }_{107}$ Cod. Theod. I2, I, 86, 87, 90, 91, 93, 94 y 98. Cfr. Barbagallo, Corrado: loc. cit., pp. 3II-3I2 y $33 \mathrm{I}$.

Io8 Barbagallo, Corrado: loc. cit., pp. 319-320, 329-331, 359-36r y 396-397. 
todo aquel que los incumpliera ${ }^{109}$. El arranque de esta visión que venía a segregar a la mujer de toda formación no religiosa está muy presente en san Pablo ${ }^{\text {IIO }}$, aunque en quien mejor se articula esta defensa de la postergación femenina de la enseñanza es en san Jerónimo, diseñador de un completo programa de educación femenino que, inspirado metodológicamente en Quintiliano, se ceñía en lo doctrinal al pensamiento paulino. Su ideal para la mujer es el de una vida ascética, a ser posible virginal, apartada completamente de la docencia y sometida a prácticas virtuosas y hogareñas, bajo la vigilancia del marido cuando no de un pastor cristiano ${ }^{\mathrm{III}}$. No sabemos si las mujeres hispanorromanas que aisladamente conocemos por los textos de aquel tiempo-Teresia, Termantia, Egeria, Poemenia, Melania- por ser todas pertenecientes a la aristocracia bajoimperial, ricas y poderosas ${ }^{112}$, cumplieron fielmente estos preceptos. Seguramente sí. La imagen que nos ofrecen las mujeres comprometidas a partir de la cuarta centuria con la herejía de Prisciliano, fieles seguidoras de éste y de sus obispos priscilianistas, nos confirman su dependencia de estos varones evangélicos ${ }^{\mathrm{I1}}$. En su comportamiento se observa cómo la mujer de la Antigüedad clásica había desaparecido, arrastrando consigo el modelo educativo romano que tanto había beneficiado su independencia. Hispania no fue una excepción. Un nuevo tiempo se abría para la educación, por y para las mujeres, en los albores medievales ${ }^{\mathrm{II}}$.

109 Vives, José: Concilios visigóticos e hispano-romanos, Barcelona-Madrid, 1953, p. I6. Concilio de Zaragoza, I (380 d. C.): «I. Ut feminae fideles a virorum alienorum coetibus separentur. Ut mulieres omnes ecclesiae catholicae et fideles a virorum alienorum lectiones et coetibus separentur, vel ad ipsas legentes aliae studio vel docendi vel discenci conveniant, quoniam hoc Apostolus iubet. Ab universis episcopis dictumest:Anathemafuturosquihanc conciliisententiamnonobservaverint».Trad.: «Quelas mujeres fieles no se mezclen en los grupos de otros hombres que no sean sus maridos. Que todas las mujeres de la Iglesia católica y bautizadas no asistan a las lecciones y reuniones de otros hombres que no sean sus maridos. Y que ellas no se junten entre sí con objeto de aprender o enseñar, porque así lo ordena el Apóstol. Todos los obispos dijeron: Sean anatemas todas aquellos que no observen esta prescripción del concilio».

${ }_{11}$ Tim., I, 2: «No permito a las mujeres enseñar a otros». Cfr. Galino, María Ángeles: loc. cit., p. $4 \mathrm{I} 2$.

iII Galino, María Ángeles: loc. cit., pp. 407-4I5. Cfr. Flecha, Consuelo: «Orden simbólico y educación en San Jerónimo», en Cerrada, Ana Isabel y Lorenzo, Josemi (eds.): De los símbolos al orden simbólico femenino (ss. IV-XVII), Madrid, 1988, pp. 7I-84.

"I2 BRAVO, Gonzalo: op. cit., p. 584.

${ }^{113}$ NúÑ̃E, Óscar: Prisciliano, priscilianismos y competencia religiosa en la Antigüedad: Del ideal evangélico a la herejía galaica, Anejos de Veleia, Vitoria-Gasteiz, 20II, pp. 42 y I24.

${ }^{114}$ LORENZO, Josemi: «La educación en la Europa altomedieval cristiana: nueve reflexiones con alguna polémica», Historia de la Educación, 26 (2007), pp. 39-63. 